\title{
重力对溶液悬浮粒子聚集过程影响的布朗动力学模拟
}

\author{
李 旭 徐升华 孙祉伟* \\ (中国科学院力学研究所, 国家微重力实验室, 北京 100190)
}

\begin{abstract}
摘要: 采用布朗动力学的计算机模拟方法, 研究重力因素对于稀溶液中悬浮粒子聚集过程的影响. 通过在计算 机模拟程序中加入和排除重力因素的影响, 对不同重力条件下的粒子团数量随时间的变化曲线进行对比研究, 得到了重力对溶液中的粒子团总数和不同大小的粒子团数量随时间变化的影响规律, 可以总结为: 在聚集阶段 初期, 重力不影响粒子的聚集; 而在聚集阶段后期, 重力加快了粒子的聚集. 同时, 从动力学分析的角度出发, 对 重力如何影响悬浮溶液中粒子聚集过程的机制也进行了更加深人的探讨.
\end{abstract}

关键词: 布朗动力学方法; 重力; 悬浮粒子; 稀溶液; 聚集; 粒子聚集团 中图分类号: O648; O641

\section{A Brownian Dynamics Simulation to Study the Influence of Gravity on the Process of Particle Coagulation in Suspension}

\author{
LI Xu XU Sheng-Hua SUN Zhi-Wei* \\ (National Microgravity Laboratory (NML), Institute of Mechanics, Chinese Academy of Sciences, Beijing 100190, P. R. China)
}

\begin{abstract}
The Brownian dynamics method was used to simulate the influence of gravity on the process of particle coagulation in a dilute solution. By including or excluding the gravitational influence in a computer simulation, the relative change in the number of aggregates with time for these two gravitational conditions could be compared. Timedependent variations of the number and size of all aggregates under the gravitational conditions were obtained. We conclude that the influence of gravity is negligible during the earlier stage of the coagulation process and that the rate of aggregation is accelerated by the gravitational force in the latter stage. The effect of gravitational force on the process of particle aggregation in suspension is discussed by using a dynamic analysis method.
\end{abstract}

Key Words: Brownian dynamics method; Gravity; Particles in suspension; Diluted solution; Coagulation; Aggregates

悬浮溶液中胶体粒子的聚集行为无论在理论研 究中还是在工业应用中都有着非常重要的意义 ${ }^{[1-6]}$. 如在环境治理方面, 研究水污染中的悬浮固体颗粒 (悬浮固体颗粒是测定工业废水污染程度的重要指 标), 研究河流携带的泥沙(江河水流中携带的大量泥 沙往往造成河道或者人海口的淤积) 以及研究空气 污染的悬浮颗粒物时(可吸人颗粒物是衡量空气污 染指数的重要指标), 都不可避免地需要研究悬浮粒 子的聚集过程以及重力沉降对聚集的影响 [7-9]. 同时,
在一些工业的生产制造中, 也有利用超重或离心等 方法, 将小粒子制备成为较大聚合物的需要. 由于粒 子聚集的研究涉及的行业和用途都比较广泛, 因此 各国的研究人员都对粒子聚集过程尤其是重力对粒 子聚集的影响机制进行了很多相关的研究 ${ }^{[1-6,10-12]}$. 一 般而言, 判断重力的影响就需要与无重力条件下的 结果进行比较. 但是, 进行无重力的实验往往需要火 箭或卫星搭载, 实验成本较高; 而计算机模拟相比而 言则很经济, 因此也就成为越来越广泛的研究手段.

Received: September 9, 2008; Revised: October 12, 2008; Published on Web: December 8, 2008.

*Corresponding author. Email: sunzw@imech.ac.cn; Tel: +8610-82544093; Fax: +8610-82544096.

国家自然科学基金(10672173, 10432060)和中国科学院知识创新工程重要方向(0518181162)资助项目

C Editorial office of Acta Physico-Chimica Sinica 
以往实验中对粒子聚集过程的研究一般采用光 散射和浊度法研究粒子的聚集行为. 近年来, 我们将 $\mathrm{T}$ 矩阵方法应用到浊度测量中, 大大提高了浊度法 测量的精度 ${ }^{[13-15]}$. 另一方面, 计算机模拟也被广泛地 用来研究粒子的聚集过程 ${ }^{[16-19]}$. 本文的主要内容就 是通过布朗动力学方法, 利用计算机模拟研究重力 因素对于粒子聚集过程的影响. 由于在计算机模拟 中可以很容易地改变重力加速度, 因此与实验研究 相比, 可以更方便而有效地认识重力对聚集过程的 影响规律。

\section{1 模拟方法和假设}

模拟微观粒子运动、相互作用与碰撞等过程的 途径很多. 典型的方法有分子动力学方法(molecular dynamics method, MD 方法) 和布朗动力学方法 (Brownian dynamics method, BD 方法) 等 ${ }^{[16-22]}$. 相对 于 MD 方法, BD 方法的优点 ${ }^{[20,21]}$ 体现在: 对于研究 的体系中存在着不同组分, 而各组分所对应的特征 时间和空间也都不同时, 由于在研究粒子的聚集过 程中仅需要关注研究对象的运动行为, 而不必考虑 与其无关的其他组分的运动细节, 此时可以采用 $\mathrm{BD}$ 方法, 将与研究对象无关的运动细节做平均化 处理, 从而更有效地进行处理和计算. 对于研究的胶 体粒子县浮溶液而言, 体系中存在着水分子和比它 们大几个数量级的胶体粒子, 两种体系对应的特征 时间和空间也相差极其悬殊. 我们研究的对象是胶 体粒子, 而不关心水分子运动和作用的细节. 因此可 以采用 $\mathrm{BD}$ 方法, 也就是将水分子对于胶体粒子运 动的贡献做平均化处理, 以布朗运动项表示. 这样, 在计算程序中就不必再采用水分子对应的较小的时 间步长而可以直接采用胶体粒子对应的相对较大的 时间步长来进行计算, 从而提高了计算效率.

在 $\mathrm{BD}$ 方法中选用的运动微分方程是朗之万方 程, 可以表示为

$$
\mathrm{d} p / \mathrm{d} t=F(t)-\gamma p(t)+R^{\prime}(t)
$$

(1)式中, $p(t)$ 代表粒子的动量, $F(t)$ 代表粒子受到的 保守力, 而 $\gamma p(t)$ 和 $R^{\prime}(t)$ 两项分别代表耗散力和随机 力. 根据朗之万方程, 在计算机模拟程序中, 第 $i$ 个 粒子在 $t$ 时刻的位置 $r_{i}(t)$ 和经过时间步长 $\Delta t$ 后的位 置 $r_{i}(t+\Delta t)$ 有如下关系 ${ }^{[20]}$ :

$$
r_{i n}(t+\Delta t)=r_{i n}(t)+\frac{D_{0}}{k_{\mathrm{B}} T} F_{i n}(t) \Delta t+\Delta r_{i n}^{G}
$$

式(2)中 $n=1 、 2 、 3$, 为笛卡尔坐标系下相互正交的三
个方向, 等式右边第二项代表由于粒子受到外力而 产生的位移, $\Delta r_{i n}^{G}$ 代表粒子在溶液中作布朗运动的 随机位移, 服从高斯分布: $\left\langle\left(\Delta r_{i n}^{G}\right)^{2}\right\rangle=2 D_{0} \Delta t$, 其中 $D_{0}$ 为 粒子在溶液中的扩散系数, 服从 Stokes-Einstein 关 系:

$$
D_{0}=k_{\mathrm{B}} T / 6 \pi \eta a_{0}
$$

式(3)中, $k_{\mathrm{B}}$ 是 Boltzmann 常数, $T$ 代表温度, $\eta$ 为溶 液的粘度, $a_{0}$ 为粒子半径. 考虑到仅有重力作用的影 响, 粒子在溶液中产生沉降的速率为 $v(t)$, 式(2)可以 进一步表示为

$$
r_{i n}(t+\Delta t)=r_{i n}(t)+v_{i n}(t) \Delta t+\Delta r_{i n}^{G}
$$

在沉降过程中, 某一时刻的粒子受到的重力 $G$ 、 浮力 $F$ 和粘滞力 $f$ 达到平衡, 得到半径为 $a_{0}$ 的球形 粒子沉降的速率 $v$ 为

$$
v=2|\Delta \rho| g a_{0}^{2} / 9 \eta
$$

在式(5)中, $g$ 为重力加速度, $\Delta \rho$ 为粒子和溶液的密 度差. 需要特别强调的是, 只有在粒子的数密度和体 积浓度都较低时, 式(5)才适用. 若粒子在溶液中数 密度较高或者粒子占据的体积分数较大, 粒子的沉 降速度就会受到临近粒子的影响 ${ }^{[20]}$. 由于在模拟体系 中粒子的数密度和体积分数非常低(远远小于 $1 \%$ ), 两粒子之间的平均距离很大, 因此临近粒子的影响 可以忽略. 通过式(4)和(5), 以及程序中采用的时间 步长, 可以进一步得到每一时间步长内粒子的沉降 距离 $L, L=v \Delta t$.

除了以上的理论和公式之外, 在计算机模拟过 程中还需引人一些假定. 首先, 假设初始存在的粒子 和后来形成的粒子团均为硬球模型, 即所有粒子和 粒子团均为球形, 并且在粒子团相互碰撞之前, 两粒 子团之间没有任何相互作用; 其次, 判断两粒子团 是否聚集的标准是, 对于半径分别为 $a_{i}$ 和 $a_{j}$ 的粒子 团, 当两者的中心距 $r_{i j}$ 满足 $r_{i j} \leqslant a_{i}+a_{j}$ 时, 即认为两 粒子团已经聚集. 新形成的粒子团内包含有 $i+j$ 个 粒子, 并且该粒子团包含的所有粒子都不会再脱离 该粒子团. 对于新形成粒子团的半径和体积, 在模拟 中采用了最为常用的融合假设 ${ }^{[23]}$, 即一个包含有 $N$ 个粒子的粒子团的半径 $a_{N}$ 可以表示为 $a_{N}=N^{1 / 3} a_{0}$, 体 积 $V_{N}$ 可以表示为 $V_{N}=N V_{0}$, 其中 $a_{0}$ 和 $V_{0}$ 代表单个粒 子的半径和体积

有了以上的模拟方法和假设, 在计算机模拟开 始之前还需给出一些准确的条件和参数. 初始时刻 生成 64000 个大小一致且半径为 $a_{0}=1.0 \mu \mathrm{m}$ 的球形 单粒子, 对应的粒子在溶液中的数密度为 $Z_{0}=2.7 \times 10^{7}$ 
$\mathrm{cm}^{-3}$, 体积分数约为 $\varphi=1.1 \times 10^{-4}$, 为极稀溶液. 粒子和 溶液的密度分别为 $\rho_{\mathrm{s}}=1.1 \mathrm{~g} \cdot \mathrm{cm}^{-3}$ 和 $\rho_{\mathrm{l}}=1.0 \mathrm{~g} \cdot \mathrm{cm}^{-3}$, 易 算得密度差为 $\Delta \rho=0.10 \mathrm{~g} \cdot \mathrm{cm}^{-3}$, 溶液的粘度系数 $\eta=$ $0.10 \mathrm{~g} \cdot \mathrm{cm}^{-1} \cdot \mathrm{s}^{-1}$, 温度取 $T=300 \mathrm{~K}$. 模拟中采用的时间 步长是 $\mathrm{d} t=0.05 \mathrm{~s}$, 共计算 200000 步, 对应粒子聚集时 间为 $t=10000 \mathrm{~s}$. 为了衡量重力对粒子聚集的影响, 在 程序中分别选用了无重力 $(g=0, g$ 指相对常重力加速 度的倍数)和普通重力 $(g=1)$ 两种条件进行比较, 不 考虑粒子间作用力和流体相互作用等其他因素的影 响, 确保两条件下的粒子聚集行为的差异完全是由 于重力的差异造成的.

\section{2 计算机模拟的结果分析}

\section{1 计算模拟与理论对比}

为了验证模拟程序的正确性, 首先需要把计算 机模拟的结果和 Smoluchowski 理论的推导值 ${ }^{[2]}$ 进 行对比. Smoluchowski 理论是在研究粒子聚集中较 为常用的一种理论, 能够通过推导得出不同时刻的 粒子团数量. 需要特别强调的是 Smoluchowski 理论 成立的条件, 包括: (1)初始溶液中的粒子均为球形 且大小一致, 即为单分散体系; (2)溶液中粒子的数 密度和浓度都非常低, 即为极稀溶液. 在本文的计算 机模拟中所使用的条件均满足上述条件, 因此可以 通过比较理论和计算机模拟的结果来验证模拟程序 的正确性.

从Smoluchowski 理论可知, 由 $N$ 个单粒子组成 的粒子团(在本文中将称为 $N$-粒子团)的数密度 $Z_{N}$ 随时间变化关系如下 ${ }^{[21]}$ :

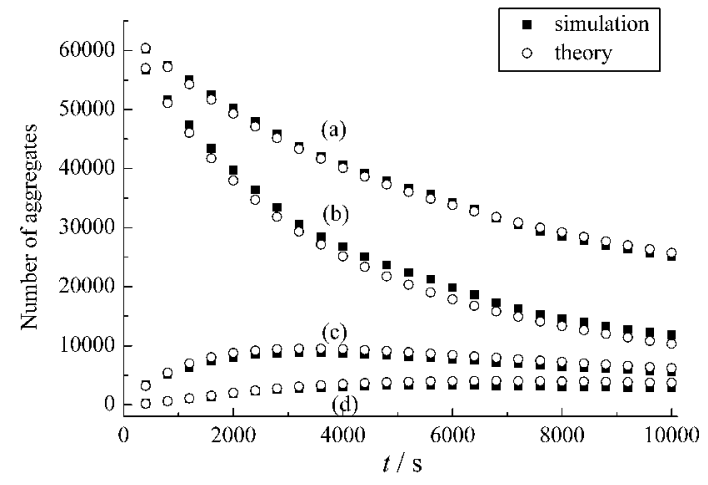

图 1 计算机模拟和 Smoluchowski 理论推导的各大小粒 子聚集团数量和粒子聚集团总数随时间变化的曲线对比

Fig.1 Comparison of the time variations of the number of different sized aggregates and all aggregates calculated from computer simulation and Smoluchowski theory

(a) all aggregates, (b) singlets, (c) doublets, (d) triplets
$Z_{N} / Z_{0}=\left(k_{\triangle} Z_{0} t\right)^{N-1} /\left(1+k_{\$} Z_{0} t\right)^{N+1}$

这里 $Z_{0}$ 是初始时刻单粒子团的数密度, $k_{\mathrm{s}}$ 为聚集速 率常数, 表示为

$k_{\mathrm{s}}=4 k_{\mathrm{B}} T / 3 \eta$

进一步得到所有不同大小粒子团的数密度之和 $\Sigma Z_{N}$ 随时间的变化关系如下:

$\Sigma Z_{N} / Z_{0}=1 /\left(1+k_{\gg} Z_{0} t\right)$

计算机模拟的结果与 Smoluchowski 的理论推 导值的比较见图 1, 图中的黑色与白色曲线分别代 表计算机模拟的结果和从 Smoluchowski 理论推导 的结果. 由上至下的四条曲线分别代表溶液中粒子 团总数, 单粒子团数量, 两粒子团数量和三粒子团数 量随时间的变化. 可以很明显的看到, 两条曲线在聚 集过程中基本重合. 同时, 经过计算机模拟得到的聚 集速率常数与 Smoluchowski 理论推导的聚集速率 常数值也极为相近, 说明本文中的计算机模拟程序 可以较为准确地反应粒子聚集的过程, 模拟过程中 所采用的一些假设和采用的一些条件也较为合理.

\section{2 模拟结果与分析}

有了以上程序计算结果和 Smoluchowski 理论 值的对比验证之后, 可以进一步将此程序中分别加 人常重力 $(g=1)$ 和无重力 $(g=0)$ 的条件, 对两条件下 的计算结果进行对比. 图 2 为计算机模拟给出的不 同重力条件下的粒子聚集曲线.

图 2 比较了有重力和无重力对聚集快慢影响的 结果. 存在重力的聚集过程不能像无重力那样可以 用 Smoluchowski 理论来解释. 从图 2 中可以看到, 在聚集初期 (前500 s内), 不同重力条件下的两条聚

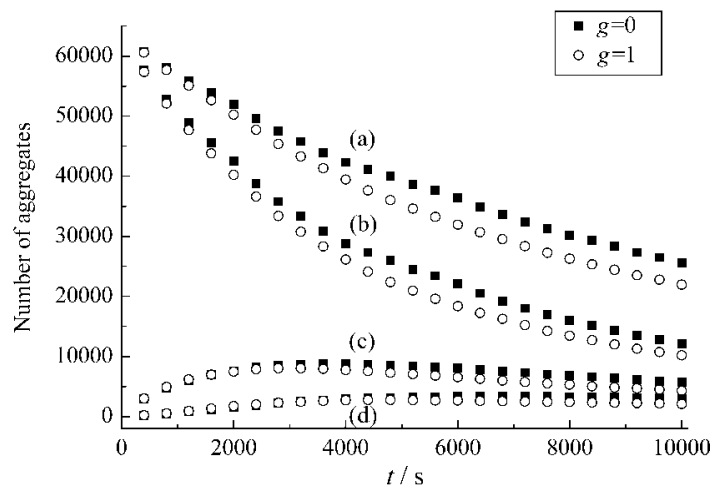

图 2 在无重力和常重力条件下计算机模拟得到的各大小 粒子聚集团数量和粒子聚集团总数随时间变化的曲线对比

Fig.2 Comparison of the time variation of the amount of different sized aggregates and all aggregates simulated under no gravity and normal gravity conditions

(a) all aggregates, (b) singlets, (c) doublets, (d) triplets 
集曲线基本重合. 造成这个现象的原因可以解释为, 由于在起始阶段的溶液中存在的粒子均为单粒子, 几乎不存在多粒子聚集团. 由于所有单粒子的大小 均一致, 通过式(5)可以计算出重力造成的各粒子的 沉降速度也一致, 因此聚集的贡献几乎都来自于粒 子的布朗运动, 而重力的存在对粒子的相互碰撞从 而导致聚集的贡献极少. 因此, 在初始阶段不同重力 条件下的聚集速率才表现为基本一致. 同时, 从图 2 中也可以明显看到, 随着聚集时间的增加(例如 $5000 \mathrm{~s}$ 之后), 粒子的聚集数量在不同的重力条件下 的差别越来越大.

可以通过理论分析来解释图 2 中的结果, 假设 初始存在任意两个粒子团分别包含 $i$ 和 $j$ 个粒子, 当两者结合成为一个新的粒子团时, 新生成的两粒 子团质量和体积均为两初始粒子团之和, 由于假设 新生成的粒子团仍保持球形, 容易算得新粒子团的 半径为单粒子的 $(i+j)^{1 / 3}$ 倍, 通过式(5)容易计算出大 粒子比小粒子的沉降速率更快, 这种速率的差异导 致在溶液中的粒子沉降过程中较大的粒子不断地超 越较小的粒子, 从而使得不同大小的粒子团在沉降 过程中得到更多的机会相互碰撞聚集, 也就是使粒 子团之间变得更容易聚集. 因而, 重力存在的条件下 粒子的聚集速率比无重力的条件下粒子的聚集速率 变得更大.

为了进一步验证这一解释, 将在 $10000 \mathrm{~s}$ 不同 大小粒子团的数量 $Z_{N}$ 进行对比. 由于此时刻产生的 大小为 $N>20$ 的 $N$-粒子团数量很少, 其数量受偶然 因素影响较大, 因此不进行比较. 这里仅对一些较有 代表性的介于 6 至 20 个粒子组成的粒子团在 $10000 \mathrm{~s}$ 时刻的数量进行对比, 如图 3 所示.

图 3 中, 当粒子团包含的单粒子数 $N$ 取 $6 、 8 、 10$ 时, 在 $10000 \mathrm{~s}$ 常重力条件下的粒子团数量显著少 于无重力条件下的同大小的粒子团数量; 当 $N$ 取 12 时, 重力条件下和无重力条件下的粒子团数量基本 一致. 当 $N$ 取 $14 、 16 、 18$ 时, 重力条件下的粒子团数 量明显多于无重力条件下同大小粒子团的数量. 可 以总结如下: 大粒子团的数量在重力条件下数量较 多, 而小粒子团数量在无重力条件下数量较多. 这是 因为重力的存在造成了聚集速率的增大, 从而导致 在同样的时间内, 在重力条件下溶液中小粒子团结 合频率更快, 从而能够形成更多的大粒子团.

通过以上的分析, 得到了重力在长时间后会加 快粒子聚集速率的结论. 为了更深人探讨重力如何

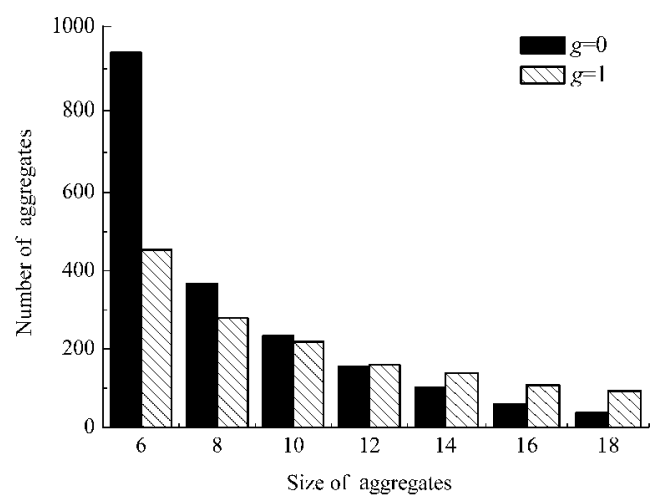

图 $310000 \mathrm{~s}$ 时在无重力和常重力条件下不同大小的 粒子团数量

Fig.3 Comparison of the number of different sized aggregates under no gravity and normal gravity conditions at $10000 \mathrm{~s}$

影响粒子的聚集过程, 需要进一步研究各大小粒子 团的数量随时间的分布, 对比在不同重力条件下不 同大小粒子团的数量随时间的变化趋势, 包括各大 小粒子团数量的变化速率与最大值, 各大小粒子团 从出现直至达到最大值所经历的时间等.

图 4 给出了在无重力和常重力条件下 4-粒子 团和 5-粒子团在溶液中数量随聚集时间的变化趋 势. 可以看出, 在常重力条件下, $N$-粒子团数量在聚 集初期增长速度较快, 增长时间较短, 达到最大值的 时间较短, 最大值较小; 而在无重力条件下, $N$-粒子 团数量在聚集初期增长速度较慢, 增长时间较长, 达 到最大值的时间较长, 最大值较大.

为了解释这一现象, 仍需要从粒子团的变化速 率着手来进行分析. 在无重力且没有外界作用的条

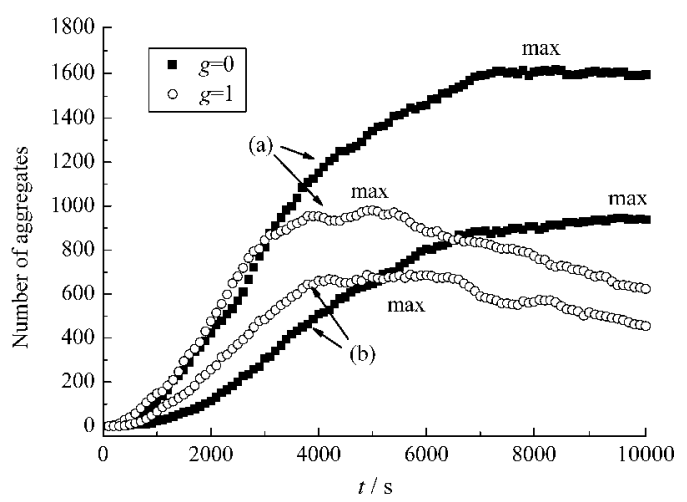

图 4 在无重力和常重力的条件下 $N$-粒子团的数量随 时间的变化曲线

Fig.4 The time variation of the number of $\mathrm{N}$-aggregates under no gravity and normal gravity conditions

(a) 4-aggregates, (b) 5-aggregates 
件下, $N$-粒子团的数量变化率可以表示为 ${ }^{[2]}$

$$
\frac{\mathrm{d} Z_{N}}{\mathrm{~d} t}=\frac{1}{2} \sum_{i=1, j=N-i}^{i=N-1} k_{i j} Z_{i} Z_{j}-Z_{N} \sum_{m=1}^{\infty} k_{m N} Z_{m}
$$

其中 $k_{i j}$ 是碰撞速率常数(the collision rate constant), 可以表示为

$$
k_{i j}=\frac{2 k_{\mathrm{B}} T}{3 \eta} \frac{\left(a_{i}+a_{j}\right)^{2}}{a_{i} a_{j}}
$$

当 $a_{i}=a_{j}$ 时, $k_{i j}$ 可以简化表示为

$$
k_{i j}=8 k_{\mathrm{B}} T / 3 \eta
$$

在式(9)中, 等号右边的第一项我们称之为形成速率 (the formation rate), 表示由比 $N$-粒子团小的两个粒 子团碰撞结合生成 $N$-粒子团的速率, 这一过程会导 致 $N$-粒子团数量的增加. 右边第二项我们称之为消 失速率(the loss rate), 表示一个 $N$-粒子团与另外任一 粒子团碰撞结合生成更大粒子团的速率, 这一过程 会导致 $N$-粒子团数量的减少. $N$-粒子团的数量变化 率取决于形成速率和消失速率, 若形成速率大于消 失速率, 则粒子团数增加, 若形成速率小于消失速 率, 则粒子团数减少.

在聚集初始阶段, $N$-粒子团 $(N>1)$ 还未出现, 仅 有比 $N$ 小的粒子团在溶液中. 此时只存在这些较小 粒子团相互碰撞结合生成 $N$-粒子团的可能性, 因此 只可能存在 $N$-粒子团的形成速率, 同时由于不存在 $N$ 粒子团, 即消失速率为零, 因而 $N$-粒子团数量逐 渐增加. 之后随着 $N$-粒子团的出现, $N$-粒子团有了 和其他各大小粒子团碰撞结合生成更大的粒子团的 可能性, 于是 $N$-粒子团的消失速率开始出现. 进而 由于 $N$-粒子团的大量出现, $N$-粒子团和其他粒子团 碰撞结合形成更大粒子团的几率一一即消失速率增 大, 而此时溶液中较小的粒子团数量由于大量碰撞 结合而减少, 即 $N$-粒子团的形成速率也开始降低. 当消失速率增加至与形成速率大小相等时, $N$-粒子 团数量达到最大值. 此后, 消失速率进一步增加, 形 成速率进一步降低, 当消失速率超过形成速率, 溶液 中粒子数量开始减少.

在出现 $N$-粒子团的初期, 由于重力的存在增加 了粒子团的碰撞几率, 因此常重力下, $N$-粒子团的形 成速率增大; 同时由于溶液中 $N$-粒子团数量较少, 因此消失速率可以忽略不计. 综合形成速率与消失 速率的影响可知, 在常重力条件下初期, $N$-粒子团数 量增长速率明显高于非重力条件下的 $N$-粒子团增 长速率. 同样的, 当溶液中的 $N$-粒子团开始明显增 多后, 由于重力的存在显著提高了 $N$-粒子团与其他
粒子团的碰撞几率, 因而消失速率显著提高. 同时由 于在重力条件下小粒子团相互碰撞结合得更为频 繁, $N$-粒子团出现初期存在的小粒子团数量也减少 得更加迅速, 因此 $N$-粒子团的形成速率也迅速降低. 由于在重力条件下, 形成速率和消失速率与无重力 条件下相比均被迅速加快, 因此在常重力条件下达 到平衡所耗费的时间的相比于无重力条件下更短. 同时, 对于在常重力条件下, $N$-粒子团数量的最大值 也相对较少的结果, 可以解释为, 由于重力条件下出 现消失速率较早, 消失速率增速较快, 形成速率的增 大后减小得也较快, 因此, $N$-粒子团数量的最大值也 相对较少.

\section{3 结 论}

通过采用布朗动力学的计算机模拟方法, 研究 了重力对于极稀溶液中悬浮粒子的聚集过程的影 响.一方面, 对重力在聚集的不同阶段对溶液中粒子 团总数的变化的影响进行了探讨, 并得出了在聚集 初期不影响粒子聚集和长期聚集过程中加快粒子聚 集的结论. 另一方面,通过计算机模拟对不同大小的 粒子团数量随时间的变化进行了研究, 对比了在重 力下与无重力环境下不同大小粒子团数量的差异, 并从粒子的聚集动力学过程出发进行了分析, 给出 了不同重力条件下粒子团数量变化的机理. 对于重 力对聚集过程的影响有了更加深人和明确的认识.

\section{References}

1 González, A. E. J. Phys. Condens. Matter, 2002, 14: 2335

2 González, A. E. Phys. Rev. Lett., 2001, 86: 1243

3 Allain, C.; Cloitre, M.; Wafra, M. Phys. Rev. Lett., 1995, 74: 1478

4 Folkersma, R.; Alois, J. G.; van Diemen, A. J.; Stein, H. N. J. Colloid Interface Sci., 1998, 206: 482

5 Folkersma, R.; Stein, H. N. J. Colloid Interface Sci., 1998, 206: 494

6 Folkersma, R.; van Diemen, A. J.; Stein, H. N. Adv. Colloid Interface Sci., 1999, 83: 71

7 Guo, W.; He, M. C.; Yang, Z. F.; Lin, C. Y.; Quan, X. C.; Wang, H. Z. Chemosphere., 2007, 68: 93

8 Müller, B.; Berg, M.; Yao, Z. P.; Zhang, X. F.; Wang, D.; Pfluger, A. Sci. Total. Environ., 2008, 402: 232

9 Zhang, M. S.; Song, Y.; Cai, X. H.; Zhou, J. J. Environ. Manage, 2008, 88: 947

10 Honig, E. P.; Roebersen, G. J.; Wiersema, P. H. J. Colloid. Interface Sci., 1971, 36: 97

11 Crocker, J. C. Phys. Rev. Lett., 1996, 106: 2837

12 Villalba, G. U.; Sucre, M. G.; Mendoza, J. T. Phys. Rev. E, 2003, 
68: 061408

13 Sun, Z. W.; Liu, J.; Xu, S. H. Langmuir, 2006, 22: 4946

14 Liu, J.; Xu, S. H.; Sun, Z. W. Langmuir, 2007, 23: 11451

$15 \mathrm{Xu}$, S. H.; Liu, J.; Sun, Z. W. J. Colloid Interface Sci., 2006, 304: 107

16 Sun, Z. W.; Chen, Z. Y. Chin. Phys. Lett., 2003, 20: 1634

17 Sun, Z. W.; Liu, J.; Xu, S. H. Chin. Phys. Lett., 2005, 22: 2119

18 Liu, J.; Sun, Z. W.; Yan, A. A. Chin. Phys. Lett., 2005, 22: 3199

19 Sun, Z. W.; Xu, S. H.; Liu, J. J. Jpn. Soc. Microgravity Appl., 2007, 24: 62

20 Allen, M. P.; Tildesley, D. J. Computer simulation of liqiuds.
Oxford: Oxford University Press, 1987: 19-32, 71-108, 147-152, $257-270$

21 Elimelech, M.; Gregory, J.; Jia, X.; Williams, R. A. Particle deposition and aggregation. Woburn, MA: Butterworth-Heinemann press, 1995: 157-202, 218-229

22 Rapaport, D. C. The art of molecular dynamics simulation. Cambridge: Cambridge University Press, 1995: 1-75, 199-266, 296-435

23 Nicolai, H.; Herzhaft, B.; Hinch, E. J.; Oger, L.; Guazzelli, E. Phys. Fluids, 1995, 7: 12 